\title{
Eletroconvulsoterapia em pacientes com transtorno afetivo bipolar: uma revisão narrativa
}

\author{
Eletroconvulsive therapy in patients with bipolar affective disorder: a narrative review
}

Terapia electroconvulsiva en pacientes con trastorno afectivo bipolar: una revisión narrativa

Matheus Jannuzzi Moreira de Mendonça ${ }^{1 *}$, Rodrigo Tavares Gonçalves ${ }^{1}$, Bruno Magalhães Gomes Macedo ${ }^{1}$, João Paulo Rossi de Meneses Vargas ${ }^{1}$, Marcos Vinícius Lino Campos ${ }^{1}$, Blenda Scalia Veneziano Esquarcio1, Vitor Augusto Lima do Vale" ${ }^{1}$, Diego Câmara Morais ${ }^{1}$, Thales Pessoa Carneiro ${ }^{1}$, Felipe Ferreira Lima ${ }^{1}$.

\section{RESUMO}

Objetivo: Revisar evidências atuais acerca da aplicação e dos benefícios da Eletroconvulsoterapia (ECT) em pacientes com transtorno afetivo bipolar. Revisão bibliográfica: Os mecanismos de ação da ECT no tratamento da bipolaridade não são totalmente elucidados e essa prática é comumente desacreditada pela própria comunidade médica, gerando descrenças quanto a sua efetividade e segurança. Todavia, há fortes correlações com o uso da terapia e a melhora significativa dos sintomas depressivos, maníacos e de estados mistos no transtorno bipolar. A taxa de resposta é, no geral, igualmente eficaz, ou superior à mediação habitual, variando conforme grupos específicos ou complexidade do quadro. A ECT é capaz, também, de reduzir a ocorrência de oscilações de humor, sendo eficaz, também, como terapia de manutenção. Uma maior resposta demonstra estar atrelada a diversos fatores, como maior velocidade da onda da ECT, posicionamento unilateral de eletrodos e idade avançada. Considerações finais: Apesar dos efeitos benéficos, a ECT não está isenta de colaterais. Mais estudos devem ser propostos para elaboração de diretrizes mais assertivas sobre o tema.

Palavras-chave: Eletroconvulsoterapia, Transtorno afetivo bipolar, Mania.

\begin{abstract}
Objective: Review current evidence regarding the application and benefits of Electroconvulsive Therapy (ECT) in patients with bipolar affective disorder. Bibliographic review: The mechanisms of action of ECT in the treatment of bipolarity are not fully elucidated and this practice is commonly discredited by the medical community itself, generating disbelief as to its effectiveness and safety. However, there are strong correlations with this therapy and significantly improvement in depressive, maniac and mixed-state symptoms. The response rate is, in general, equally efficient, or superior to the usual mediation, varying according to specific groups or complexity of the condition. ECT is also capable of reducing the occurrence of mood swings, being also effective as maintenance therapy. A greater efficiency seems to be linked to factors, such as higher wave velocity, unilateral electrode positioning and advanced age. Final considerations: Despite the beneficial effects, ECT is not exempt from collaterals. Further studies should be proposed to develop more assertive guidelines on the topic.
\end{abstract}

Keywords: Electroconvulsive therapy, Bipolar disorder, Mania.

${ }^{1}$ Pontifícia Universidade Católica de Minas Gerais (PUC Minas), Belo Horizonte - MG.

*E-mail: matheusjannuzzi95@hotmail.com 


\section{RESUMEN}

Objetivo: Revisar la evidencia actual sobre la aplicación y los beneficios de la Terapia Electroconvulsiva (TEC) en pacientes con transtorno afectivo bipolar. Revisión bibliográfica: Los mecanismos de acción de la TEC en el tratamiento de la bipolaridad no están completamente aclarados y esta práctica es comúnmente desacreditada por la comunidad médica, generando incredulidad en cuanto a su efectividad y seguridad. Sin embargo, existen fuertes correlaciones con el uso de la terapia y una mejora significativa en los síntomas depresivos, maníacos y de estado mixto. La tasa de respuesta es, en general, igualmente eficaz o superior a la mediación habitual, variando según grupos específicos o la complejidad de la situación. La TEC también es capaz de reducir la aparición de cambios de humor, siendo también eficaz como terapia de mantenimiento. Se muestra que una mayor respuesta está relacionada con varios factores, como una mayor velocidad de la onda ECT, la colocación de electrodos unilaterales y edad avanzada. Consideraciones finales: A pesar de los efectos beneficiosos, la TEC no está exenta de garantías. Se deberían proponer más estudios para desarrollar pautas más asertivas sobre el tema.

Palabras clave: Terapia electroconvulsiva, Trastorno afectivo bipolar, Manía.

\section{INTRODUÇÃO}

O Transtorno Afetivo Bipolar (TAB) é uma patologia psiquiátrica grave definida por oscilações do humor, com períodos de mania ou hipomania e períodos de depressão, com recuperação entre os episódios (HILTY DM, et al., 2006; SHAH N, et al., 2017). A mania é caracterizada por uma extrema felicidade, comportamento extrovertido ou irritabilidade, associados a uma crescente energia. A hipomania, por sua vez, assemelha-se à mania, em menor intensidade e com menor prejuízo ao sujeito. Por fim, a depressão é caracterizada por sintomas como tristeza e anedonia, relacionados à redução do estado de humor (SHEN YC, 2018). Essas alterações levam, ao longo do tempo, à perda funcional, cognitiva e de qualidade de vida (SHEN YC, 2018; PERUGI G, et al., 2017). Estima-se que a prevalência de transtornos do espectro bipolar possa atingir até $2,4 \%$ da população mundial, afetando pessoas com a média de idade entre 20 e 30 anos, em sua maioria (ELIAS A, et al., 2020).

Diversas opções terapêuticas medicamentosas estão disponíveis para o controle da sintomatologia, porém, não é incomum a ocorrência de refratariedade à essas terapias (ELIAS A, et al., 2020). Destaca-se como alternativa a Eletroconvulsoterapia (ECT), método terapêutico o qual é, constantemente, desmoralizado por leigos e pela própria comunidade médica (PEREIRA D, et al., 2020). Soma-se a forma como a ECT é mostrada em filmes e obras teatrais, impactando a população, em geral. Um estudo de Walter G, et al. (2002) descreveu que um terço dos estudantes de medicina observados, os quais, em teoria, têm certo conhecimento sobre a temática, expressaram opiniões negativas quanto ao uso da ECT após assistirem cenas desse procedimento em filmes.

A ECT é um eficaz método para tratamento da clínica de depressão unipolar e bipolar, mania, esquizofrenia, transtorno esquizoafetivo, além de doenças neurais, como doença de Parkinson e síndrome neuroléptica maligna, entre outras patologias, em casos de resistência à intervenção medicamentosa inicialmente proposta ou na necessidade de intervenção rápida da sintomatologia (MEDDA P, et al., 2020; WEISS A, et al., 2019). De acordo com diversas das diretrizes da terapia com eletrochoque, ela não é considerada uma terapia de primeira linha, porém, há um consenso na psiquiatria para o uso precoce de ECT, principalmente em quadros graves e com risco de vida iminente ao paciente, como agressividade acentuada ou planejamento suicida (PARKER GB, et al., 2017; PERUGI G, et al., 2017).

A resistência quanto ao uso desse tratamento é, em grande parte, devido aos efeitos adversos somáticos e, principalmente, às alterações cognitivas. O risco de comprometimento neurocognitivo mediado por ECT está associado a fatores do próprio paciente, como idade, morbidades médicas e reserva cognitiva, além de condições do próprio tratamento, tais como a colocação de eletrodos, parâmetros de estimulação, número de sessões e a frequência de suas realizações (ABBOTT CC, et al., 2019). 
Dentre os acometimentos neurocognitivos ressalta-se a possibilidade da ocorrência de delirium que pode perdurar por dias ou algumas semanas. À curto prazo, observa-se uma amnesia anterógrada como principal efeito colateral da ECT, que tende, da mesma forma, à resolução ao longo dos dias (DONAHUE AB, 2000).

No longo prazo, o efeito mais comum é o de alteração de memória persistente. Apesar disso, estima-se que a função cognitiva geral do paciente não se altera ou melhore com o passar do tempo, decorrente da melhora sintomática de seu quadro neuropsiquiátrico inicial (WONG VK, et al., 2019). Segundo Devanand DP, et al. (1994) e Salleh MA, et al. (2006), não há associação entre a terapia de eletrochoque e a ocorrência de lesões estruturais cerebrais em humanos. O método não possuí contraindicações absolutas. $O$ estado de gravidez, inclusive, é seguro para realização do procedimento, principalmente no segundo e terceiro trimestres, apresentando alta eficácia (ROSE S, et al., 2020).

A ECT demonstra-se uma alternativa viável para o tratamento de alguns transtornos psiquiátricos. No entanto, até mesmo na comunidade médica, ainda existe um estigma sobre essa terapêutica, de maneira que a falta de informação e divulgação, tornam essa terapia uma possibilidade pouco explorada. $O$ dado estudo, portanto, objetiva reunir a atual evidência existente sobre a aplicação e os benefícios da ECT para pacientes com TAB.

\section{REVISÃO BIBLIOGRÁFICA}

O mecanismo de ação da ECT que exerce seus efeitos terapêuticos ainda não é totalmente esclarecido, apesar de haver um consenso de que a eficácia da terapia deriva da indução de crises convulsivas generalizadas, de forma controlada e comprimento específico pré-determinado (PINNA M, et al., 2018).

Analisando o estudo de Lv Q, et al. (2020), foi constatado que, apesar de incertezas, a fisiopatologia do transtorno bipolar parece estar associada ao estresse oxidativo cerebral. O composto orgânico melondialdeído (MDA), importante marcador de estresse oxidativo, encontrava-se aumentado em pacientes com diagnóstico de TAB, nas fases maníaca e depressiva da doença $(p=0,001)$. Após 6 semanas de tratamento com ECT, o nível de MDA em pacientes responsivos reduziu, comparado aos níveis prévios $(5,09$ $1,80$ vs $3,84-1,12, p=0,001)$. Mecanismos relacionados com o limiar convulsivo também demonstram possível associação com a melhora sintomática do transtorno bipolar. A ECT possuí propriedades anticonvulsivantes, decorrentes de um aumento progressivo do limiar de convulsão do paciente, o que está correlacionado com a melhora dos sintomas maníacos. O retorno do limite convulsivo basal da pessoa, também está associado às recidivas desses sintomas (ELIAS A, et al., 2020).

Não somente atrelada à redução de marcadores bioquímicos, a eficácia da ECT depende, também, do posicionamento dos eletrodos. A colocação de eletrodos em posição bitemporal é a opção mais eficaz e rápida relacionada ao estado depressivo. Em contrapartida, essa opção demonstra ter o maior comprometimento neurocognitivo. Investigações recentes sugerem que a colocação de eletrodos focais em regiões específicas tem potencial de otimizar os resultados clínicos do tratamento para transtorno bipolar, bem como reduzir o comprometimento neural (ABBOTT CC, et al., 2019).

Características individuais também estão associadas à resposta do paciente à ECT. Curiosamente, pacientes mais velhos apresentam, em sua maioria, melhores respostas clínicas que os mais jovens, à medida que a faixa etária entre os 16 e 30 anos é detentora dos piores índices. Dados demonstram uma relação significativa entre a idade e a resposta à ECT em modelos univariados (OR 1,02, IC 95\%, 1,01-1,03, $p=$ 0,001 ) e multivariados (OR 1,011, IC 95\%, 1,001-1,021, $p=0,034$ ) (POPIOLEK K, et al., 2019). Diferenças na eficácia da resposta dessa terapia entre os sexos, por sua vez, não foram evidenciadas (PINNA M, et al., 2018).

Os possíveis efeitos colaterais cognitivos decorrentes da ECT são os principais limitantes ao uso mais frequente dessa terapêutica (ELIAS A, et al., 2016; ABBOTT CC, et al., 2019). A fim de reduzir o comprometimento neural, há a disponibilidade de uma modalidade da ECT na qual utiliza-se pulsos breves, em comparação com o padrão (SHEN YC, 2018). Essa alternativa é denominada Ultrabrief Eletroconvulsive Therapy (RUB-ECT), na qual utilizam-se pulsos de $0,3 \mathrm{~ms}$ ou menos durante o procedimento. Os pulsos em ECTs habituais variam de $0,5 \mathrm{~ms}$ a $1,5 \mathrm{~ms}$. Estudos apontam que a RUB-ECT é substancialmente menos adversa ao paciente, para efeitos de curto e longo prazo (ELIAS A, et al., 2016). 
Quanto à eficácia, a RUB-ECT apresenta altas taxas de remissão de sintomas entre $70 \%$ e $100 \%$, principalmente com a colocação unilateral direita do eletrodo. Essa colocação específica do eletrodo, em comparação com a colocação bitemporal da ECT convencional, apresenta maior eficácia e menor número médio de sessões para tratamento (ELIAS A, et al., 2020). Na maioria das diretrizes mundiais a respeito do tratamento do $T A B$, preconiza-se a utilização de terapia farmacológica como primeira opção terapêutica (PARKER GB, et al., 2017). Apesar de ser considerada o método de escolha, a monoterapia medicamentosa para estados de mania apresenta resposta em cerca de $40 \%$ a $60 \%$ dos pacientes (ELIAS A, et al., 2020).

Apesar do aumento da disponibilidade e acessibilidade a medicamentos, a parcela de pacientes com transtorno bipolar que permanece refratária ao tratamento farmacológico é uma das responsáveis diretas da elevação da taxa de morbidade e de mortalidade da doença. A dosagem do medicamento, em concomitância às associações entre os diversos fármacos utilizados, gera preocupações quanto à resistência ao tratamento, ao aumento da taxa de troca, o efeito de ciclagem rápida, à não aderência terapêutica e ao aumento dos efeitos colaterais correspondentes às interações medicamentosas. Uma falha terapêutica bem documentada, por sua vez, pode levar meses ou anos, o que pode prolongar, também, o sofrimento do paciente com os sintomas experienciados (PERUGI G, et al., 2017; PATEL RS, et al., 2019).

Reforça-se, portanto, ao evidenciar as limitações da farmacoterapia, a necessidade de um consenso entre diretrizes, possibilitando a padronização dos critérios para utilização da ECT no transtorno bipolar, com base em dados estatísticos de alta relevância (PARKER GB, et al., 2017).

Embora seja potencialmente lesiva, a terapia de eletrochoque demonstra ser extremamente eficiente no transtorno bipolar, em fases maníacas, depressivas e mistas, obtendo resultados em 75,0\%, 68,1\% e 72,9\% para as respectivas fases, após realização de 3 sessões por semana, por tempo variado, em pacientes bipolares resistentes à terapia medicamentosa (PERUGI G, et al., 2017). Quando analisada a população de adultos com transtornos do humor resistentes, observou-se uma taxa de sucesso variando entre $60 \%$ e $80 \%$ (WEINER RD e RETI IM, 2017).

Em pacientes resistentes à terapêutica convencional, há uma maior ocorrência de resultados positivos quanto à remissão da sintomatologia após 6 semanas sob uso de ECT, em comparação com a continuidade do tratamento com fármacos, no mesmo período $(73,9 \%$ vs $35,0 \%$, NNT = 3) (SHEN YC, 2018). Segundo o estudo de Atay ÖC, et al. (2019), a melhora clínica de pacientes bipolares ao serem submetidos à ECT não é somente identificada pelos observadores externos à terapia. Grande parte dos pacientes consideram a terapia benéfica para si próprios e até $84 \%$ desses pacientes relatam melhora na qualidade de vida após a realização de 7 a 10 sessões de choque.

Em um acompanhamento longitudinal de 57 semanas após o uso da ECT, constatou-se que até $93 \%$ dos pacientes obtêm resposta, ao menos, parcial à terapêutica por cerca de $90 \%$ do período acompanhado. Desses, até $73 \%$ preencheram critérios para remissão completa do quadro. Houve, nesse seguimento, maior remissão em pacientes em estado depressivo, comparados aos em estado misto $(83,3 \%$ vs $61,8 \%, p=0,043)$ (MEDDA P, et al., 2020). Paralelamente, dados do estudo de Popiolek K, et al. (2019) demonstram que aproximadamente $80 \%$ de pacientes submetidos à ECT obtêm resposta terapêutica para sintomas depressivos bipolares, enquanto a piora desses sintomas após 1 semana da terapia ocorre em menos de $1 \%$ das pessoas. Em contrapartida, alguns estudos não evidenciam diferenças de taxas de remissão da doença em comparação à terapia farmacológica, fato que pode ser explicado pela interrupção precoce do tratamento com eletrochoque, antes de atingir o seu benefício total (SHEN YC, 2018).

Ademais, a ECT pode o tempo de hospitalização em pacientes com TAB, entre outras patologias psiquiátricas, tendo sido necessárias, em um estudo longitudinal, cerca de 9,4 ( \pm 2$)$ sessões para resolução de mania bipolar (ITTASAKAUL P, et al., 2020). Bodicherla KP, et al. (2020) infere que em pacientes bipolares internados e manejados com ECT possuem tempo de permanência mais longo, devido ao fato dessa terapia ser utilizada, classicamente, como último recurso para tratamento. $O$ início mais precoce, nos primeiros 7 dias de internação, da terapia de eletrochoque pode reduzir o tempo de permanência em cerca de 14 dias, impactando beneficamente nos custos de gerenciamento de pacientes internados. Ressalta-se que adultos acima de 50 anos possuíram, nesse trabalho, chances 2,4 vezes maiores de internação por mais de 16 dias (IC 95\%, 2,06-2,87) (BODICHERLA, et al., 2020). 
Algumas pesquisas apontam que, em fase maníaca, a terapia com eletrochoque apresenta resultados equivalentes ou superiores, em comparação com o uso de carbonato de lítio, haloperidol e clorpromazina, entre outros estabilizadores de humor e antipsicóticos (WONG VK, et al., 2019). Entretanto, resultados conflitantes são demonstrados em estudos menores, os quais não demonstraram superioridade da ECT em comparação com o lítio na melhora de mania aguda em indivíduos hospitalizados (PINNA M, et al., 2018).

O uso da ECT combinada, também, a outro fármaco é uma prática corriqueira na atuação médica. Estimase que pacientes em fase de mania podem apresentar menos efeitos adversos aos antipsicóticos e estabilizadores de humor, em contraste com os pacientes em fases depressivas da bipolaridade. Dessa forma, a ocorrência de delírio em pacientes tratados com a combinação de lítio e ECT é substancialmente maior em pacientes com depressão, unipolar ou bipolar, enquanto a ocorrência de delírio após esse tratamento em fases maníacas é próxima ao zero. Em concomitância, a ocorrência de delírio em tratamento isolado com ECT demonstra ser significantemente menor do que no tratamento combinado (PATEL RS, et al., 2020). Associada à clorpromazina, a terapia de choque mostra-se, paralelamente, superior em remissão da clínica maníaca, ao ser comparada com o uso do medicamento isoladamente (PINNA M, et al., 2018).

O estado misto, por sua vez, é configurado como uma combinação de sintomas depressivos e maníacos ao mesmo tempo. Nos pacientes que apresentam essa configuração de alteração do humor, o tratamento farmacológico é desfavorável, devido à apresentação clínica mais complexa. Nesse contexto, destacam-se outras formas terapêuticas, como a ECT. Dados propõem que a ECT é eficaz para melhora clínica dos sintomas do estado misto, sendo responsiva em até $72,9 \%$ dos pacientes. Curiosamente, naqueles não responsivos ao tratamento, o tempo médio de duração do episódio sintomático pode ser maior doque nos responsivos (13,49 vs $7,56, P=0,001)$, assim como a taxa de episódios superiores a 1 ano $(38,1 \%$ vs $20,4 \%$, $P=0,037)$ (PERUGI G, et al., 2017).

Medda P, et al. (2020) aponta números semelhantes para melhora de sintomas depressivos em episódios isolados e episódios mistos, apesar de não observar diferenças entre a ocorrência da sintomatologia maníaca e hipomaníaca em ambos os grupos, reforçando a afirmativa de maior complexidade do tratamento dos estados mistos.

A curto prazo, a ECT se mostra uma interessante alternativa para tratamento. Ao contrário da farmacoterapia, seu uso costuma ser interrompido após remissão do episódio em questão (MEDDA P, et al., 2020). Sendo assim, apesar da ausência de documentação da falha com a terapia medicamentosa, há um grande costume na prática clínica, de uso da ECT em situações nas quais há uma rápida necessidade de remissão dos sintomas do paciente, devido a um risco acentuado de vida. Tais situações podem ser expressas por uma iminência de suicídio, quadros de desnutrição e desidratação, episódios graves de mania e depressão, catatonia, entre outros (PERUGI G, et al., 2017).

Diretrizes de alguns países, como Estados Unidos da América, Inglaterra e Canadá, listam a ECT como primeira linha de tratamento, quando há risco de autoextermínio. O rápido efeito da terapia para resolução sintomática pode se traduzir em um potencial protetor contra o suicídio. Todavia, dados não apontam correlação estatística significante da ECT para um efeito protetor ao autoextermínio nos episódios de mania bipolar (HR 1,048, IC 95\% , 0,298-1,853, p = 0,538) ou episódios mistos (HR 0,976, IC 95\%, 0,647-1,015, p $=0.126)$, apesar desse efeito preventivo ser observado nos episódios depressivos bipolares (HR 0,805, IC 95\%, 0,514-0,987, $p=0.046$ ) (LIANG CS, et al., 2018).

O tratamento da bipolaridade nos períodos de gestação e puerpério configuram, também, outra situação específica na qual há certa dificuldade de manejo medicamentoso. No período pós-parto sobressai-se a ocorrência de episódios depressivos, em comparação aos maníacos e hipomaníacos. Na ocorrência da depressão, estima-se se que até $22,6 \%$ dessas mulheres apresentam triagem para bipolaridade (CLARK CT, WISNER KL, 2018). Apesar de baixos números absolutos, o risco de desenvolvimento de surtos psicóticos relacionados com alterações do humor é cerca de 23 vezes maior no período até 4 semanas após o parto, comparado a qualquer outro período da vida da mulher, com maior ocorrência entre o $3^{\circ}$ e $10^{\circ}$ dia após 0 nascimento do filho (BERGINK V, et al., 2016). 
Medicações de primeira linha para o transtorno bipolar, como lítio, carbamazepina e a risperidona estão associadas com riscos de malformações fetais. Nesse cenário, a ECT passa a ser uma importante e segura alternativa para tratamento, principalmente na ocorrência de resistência farmacológica e necessidade de interagir medicamentos, visto que o TAB não tratado está intimamente relacionado com eventos adversos ao feto e durante o parto (CLARK CT, WISNER KL, 2018).

Outra população de extrema relevância para o uso da ECT é a geriátrica, devido à alta prevalência de diversos distúrbios crônicos nesses grupos e ao maior risco de efeitos adversos à medicação (BODICHERLA KP, et al., 2020). Nos idosos, a taxa de remissão de sintomatologia depressiva pode ser 3,04 vezes superior com a terapia de eletrochoque, comparada à medicação isolada $(63,8 \%$ vs $33,3 \%, p=0,008)$, enquanto para mania, as taxas de resposta demonstram-se semelhantemente superiores, chegando a $80 \%$. Com relação à ocorrência de episódios mistos, eles são mais prevalentes em idades mais avançadas. O difícil manejo medicamentoso nessas situações, reforça uma indicação para utilização da ECT nesse grupo (MEYER JP, et al., 2019).

Paralelamente, a utilização da ECT em adolescentes demonstra ser igualmente segura, obtendo taxas de eficiência terapêutica de até $93 \%$ em mania. A presença de comorbidades como transtornos de personalidade, transtornos dissociativos, transtorno por uso de substâncias e déficits cognitivos não contraindica essa prática terapêutica. Embora a eficácia de tratamento seja reduzida na presença dessas comorbidades $(p=0,001)$, essa resposta permanece significativa $(p<0,001)$ (KARAYAĞMURLU A, et al., 2020).

Não somente a curto prazo, a ECT pode ser utilizada como terapia de manutenção. Evidências sugerem que a terapia de eletrochoque reduz significativamente a ocorrência de episódios de oscilação de humor, aumentando os períodos eutímicos entre as crises (ELIAS A, et al., 2021). Dados apontam, também, que grupos tratados com ECT associada à farmacoterapia em períodos de manutenção, são superiores à manutenção farmacológica isolada, principalmente em episódios depressivos (LIANG CS, et al., 2018).

Apesar das evidências favoráveis ao uso da ECT, a terapêutica não está isenta de efeitos colaterais e potencial maléfico ao paciente. Mesmo com uma considerável resposta clínica, até cerca de $70 \%$ dos pacientes podem relatar esquecimento após 1 mês da terapia de eletrochoque, sendo mais frequente o relato nos que apresentam sintomas maníacos $(p=0,02)$. Cefaleia pode ser relatada em aproximadamente $56 \%$ desses pacientes e até 34\% descrevem o procedimento como doloroso, apesar da indução anestésica (ATAY ÖC, et al., 2019).

Características individuais dos pacientes e o método de aplicação da ECT são intimamente associados com a ocorrência ou não desses efeitos (ABBOTT CC, et al., 2019). Ondas de pulso ultrarrápido e colocação unilateral de eletrodo à direita, por exemplo, foram associados a uma redução significante de comprometimento cognitivo, como ocorrência de amnésia retrógrada, ao longo de 6 meses após tratamento (SHEN YC, 2018). Além dos clássicos efeitos esperados decorrentes da convulsão provocada, destaca-se a possibilidade de mania induzida, de forma iatrogênica, no tratamento de depressão bipolar (THOMAS RK, et al., 2018). É, também, necessária cautela quanto à utilização de altos números de sessões de ECT, devido ao risco de uma necessidade futura de cargas cada vez maiores para o tratamento do TAB, principalmente em refratários ao eletrochoque (NAHDIYA S, SYUKRI C, 2018).

\section{CONSIDERAÇÕES FINAIS}

A bibliografia utilizada sugere que a ECT é uma alternativa para sintomatologia bipolar, em períodos depressivos, maníacos e mistos, principalmente mediante uma necessidade de rápida resolução. Todavia, não há um consenso global para as indicações de utilização da terapia de eletrochoque, apesar dos estudos analisados apontarem sua eficácia e segurança na prática médica. Mais pesquisas devem ser realizadas, a fim de contribuir com a elaboração de diretrizes mais claras para as indicações da ECT, tornando a recomendação dessa prática mais favorável ao uso clínico. 


\section{REFERÊNCIAS}

1. ABBOTT CC, et al. Electroconvulsive therapy electrode placement for bipolar state-related targeted engagement. Int. J. Bipolar Disord., 2019; 7(1): 11.

2. ATAY ÖC, et al. Satisfaction and attitude of bipolar patients regarding electroconvulsive therapy: modified or unmodified. Nord. J. Psychiatry, 2020; 74(2): 131-137.

3. BERGINK V, et al. Postpartum Psychosis: Madness, Mania, and Melancholia in Motherhood. Am. J. Psychiatry, 2016; 173(12): 1179-1188.

4. BODICHERLA KP, et al. Predictors for Extending Hospitalization Stay in Electroconvulsive Therapy Recipients With Bipolar Disorder, Manic Episodes. Cureus, 2020; 12(6): e8832.

5. CLARK CT, WISNER KL. Treatment of Peripartum Bipolar Disorder. Obstet. Gynecol. Clin. North. Am., 2018; 45(3): 403-417.

6. DEVANAND DP, et al. Does ECT alter brain structure? Am. J. Psychiatry, 1994; 151(7): 957-970.

7. DONAHUE AB. Electroconvulsive therapy and memory loss: a personal journey. J. ECT, 2000; 16(2): 133-143.

8. ELIAS A, et al. Ultrabrief Electroconvulsive Therapy for Mania: Data From 11 Acute Treatment Courses. The journal of ECT, 2016; 32(4): 270-272.

9. ELIAS A, et al. Electroconvulsive Therapy in Mania: A Review of 80 Years of Clinical Experience. Am. J. Psychiatry, 2020; 178(3): 229-239.

10. HILTY DM, et al. A review of bipolar disorder in adults. Psychiatry (Edgmont), 2006; 3(9): 43-55.

11. ITTASAKAUL P, et al. Number of Electroconvulsive Therapy Sessions required for Thai Psychiatric Patients: a Retrospective Study. Neuropsychiatr Dis. Treat., 2020; 16: 673-679.

12. KARAYAĞMURLU A, et al. Efficacy and Safety of Electroconvulsive Therapy in Adolescents: A Retrospective Chart Review Study from Turkey. J. ECT, 2020; 36(1): 54-59.

13. LIANG CS, et al. Superior anti-suicidal effects of electroconvulsive therapy in unipolar disorder and bipolar depression. Bipolar Disord., 2018; 20(6): 539-546.

14. LV Q, et al. Disturbance of Oxidative Stress Parameters in Treatment-Resistant Bipolar Disorder and Their Association with Electroconvulsive Therapy Response. Int. J. Neuropsychopharmacol., 2020; 23(4): 207-216.

15. MEDDA P, et al. Naturalistic Follow-Up in Bipolar patients after successful Electroconvulsive Therapy. Journal of Affective Disorders, 2020; 271: 152-159.

16. MEYER JP, et al. Electroconvulsive Therapy in Geriatric Psychiatry: A Selective Review. Clin. Geriatr. Med., 2020; 36(2): 265-279.

17. NAHDIYA S, SYUKRI C. Post-electrocution persistent manic-depressive syndrome: a unique diagnostic syndrome and a red flag against electroconvulsive therapy? Med. J. Malaysia, 2018; 73(6): 441-442.

18. PARKER GB, et al. Is there consensus across international evidence-based guidelines for the management of bipolar disorder? Acta. Psychiatrica Scandinavica, 2017; 135(6): 515-526.

19. PATEL RS, et al. Combination of lithium and electroconvulsive therapy (ECT) is associated with higher odds of delirium and cognitive problems in a large national sample across the United States. Brain Stimul., 2020; 13(1): 15-19.

20. PATEL RS, et al. Electroconvulsive Treatment Utilization for the Inpatient Management of Severe Manic Episodes of Bipolar Disorder. The Journal Of Ect, 2019; 35(3): 195-200.

21. PEREIRA D, et al. Eletroconvulsivoterapia: Um Problema de Literacia em Saúde [Electroconvulsive Therapy: A Health Literacy Problem]. Acta. Med. Port., 2020; 33(7-8): 529.

22. PERUGI G, et al. The Role of Electroconvulsive Therapy (ECT) in Bipolar Disorder: Effectiveness in 522 Patients with Bipolar Depression, Mixed-state, Mania and Catatonic Features. Curr. Neuropharmacol., 2017; 15(3): 359-371.

23. PINNA $M$, et al. Clinical and biological predictors of response to electroconvulsive therapy (ECT): a review. Neuroscience Letters, 2018; 669: 32-42.

24. POPIOLEK K, et al. Electroconvulsive therapy in bipolar depression - effectiveness and prognostic factors. Acta. Psychiatr. Scand., 2019; 140(3): 196-204.

25. ROSE S, et al. Electroconvulsive Therapy in Pregnancy: Safety, Best Practices, and Barriers to Care. Obstet. Gynecol. Surv., 2020; 75(3): 199-203.

26. SALLEH MA, et al. Eletroconvulsoterapia: critérios e recomendações da Associação Mundial de Psiquiatria. Rev. psiquiatr. clín., 2006; 33(5): 262-267.

27. SHAH N, et al. Clinical Practice Guidelines for Management of Bipolar Disorder. Indian J. Psychiatry, 2017; 59(1): S51S66.

28. SHEN YC. Treatment of acute bipolar depression. Tzu Chi Medical Journal, 2018; 30(3): 141-147.

29. THOMAS RK, et al. Treating electroconvulsive therapy-induced mania with more electroconvulsive therapy: evidence for electroconvulsive therapy as the ultra-mood stabilizer. Sage Open Medical Case Reports, 2018; 6: $2050313 \times 18799236$.

30. WALTER G, et al. Medical student knowledge and attitudes regarding ECT prior to and after viewing ECT scenes from movies. J. ECT, 2002; 18(1): 43-46.

31. WEINER RD, RETI IM. Key updates in the clinical application of electroconvulsive therapy. Int. Rev. Psychiatry, 2017; 29(2): 54-62.

32. WEISS A, et al. Royal Australian and New Zealand College of Psychiatrists professional practice guidelines for the administration of electroconvulsive therapy. Aust. N. Z. J. Psychiatry, 2019; 53(7): 609-623.

33. WONG VK, et al. Effectiveness and Cognitive Changes With Ultrabrief Right Unilateral and Other Forms of Electroconvulsive Therapy in the Treatment of Mania. The Journal Of Ect, 2019; 35(1): 40-43. 